\title{
Search for non-equilibrium thermoelectrics
}

\author{
Ichiro Terasaki \\ Department of Applied Physics, Nagoya University, Nagoya 464-8602, Japan \\ Ryuji Okazaki \\ Department of Physics, Faculty of Science and Technology, Tokyo University of Science, Noda 278-8510, Japan \\ Hiromichi Ohta \\ Research Institute for Electronic Science, Hokkaido University, Sapporo 001-0020, Japan
}

\begin{abstract}
Since thermoelectrics is an energy-conversion technology from heat flow to electrical flow, or vice versa, it is essentially a non-equilibrium phenomenon in the sense that energy and particle flows are involved. We have recently focused on this feature in order to overcome theoretical predictions of the upper limit of the conversion efficiency in thermal equilibrium conditions. One trial is the thermoelectric phenomena in the range of non-ohmic conduction. The second approach is the thermoelectric phenomena shown by the photo-doped carriers.
\end{abstract}

Keywords: thermoelectrics, non-equilibrium steady state, strongly correlated electrons, photo-doping

\section{Introduction}

Thermoelectric phenomena in solids have been extensively investigated from both scientific and technological interests [T]. They are tightly related to thermodynamic 5 properties of conducting electrons, known as the transport entropy in solids. At the same time, they offer an efficient way to directly convert heat into electric energy, or vice versa, which enables us to recover electric power from waste heat and to refrigerate objects electronically.

10 Such energy conversion technology is called thermoelectrics $[2,[3]$. For the last decade, we have proposed and investigated oxide thermoelectrics: thermoelectric energy conversion using oxide materials [4].

Materials' performance for thermoelectric energy conversion is characterized by the figure of merit $Z=S^{2} / \rho \kappa,{ }^{4}$ where $S$ the Seebeck coefficient, $\rho$ the resistivity, and $\kappa$ is the thermal conductivity. When the temperature dependence of $S, \rho$ and $\kappa$ can be neglected, the energy conversion efficiency is determined by the dimensionless fig-

20 ure of merit $Z T$, where $T$ is the absolute temperature. Accordingly, a good thermoelectric material requires low conductivity, large Seebeck coefficient, and low thermal conductivity simultaneously. Since the three parameters are related to one another, a large $Z T$ value is difficult to

25 find in existing materials, and $Z T=1$ is a practical goal ${ }^{50}$ for applications. In fact, reliable calculations of materials parameters do not give $Z T>3$.

Email address: terra@cc.nagoya-u.ac.jp (Ichiro Terasaki)
We should emphasize that such theoretical predictions are based on equilibrium states, and there remains a possibility that the thermoelectric performance may go beyond theoretical limitations when the system is out of equilibrium. For example, Casati et al. [5] theoretically proposed a model ballistic system, in which the $Z T$ value exceeds ten against properly chosen parameters. In this article, we show our preliminary results for two types of non-equilibrium thermoelectrics. One is the thermoelectric properties in large current density, and the other is the thermoelectrics using photo-doped carriers.

\section{Non-equilibrium states}

The word of non-equilibrium covers a wide range of states, because it means any states that are not in thermal equilibrium. In equilibrium states, macroscopic thermodynamic variables are uniquely determined by a small number of macroscopic variables, the relationship of which is given by the thermodynamics. Even when the system is slightly out of equilibrium, the response of the system can be quantitatively predicted from the original equilibrium state through the fluctuation-dissipation theorem. Thus the non-equilibrium states we discuss in this article are those far from thermal equilibrium, where conventional thermodynamics and statistical physics are not justified to apply.

Transient phenomena at short time scale are most remarkable non-equilibrium states [6]. Thanks to recent de55 velopment in pulsed laser technique, we can observe re- 


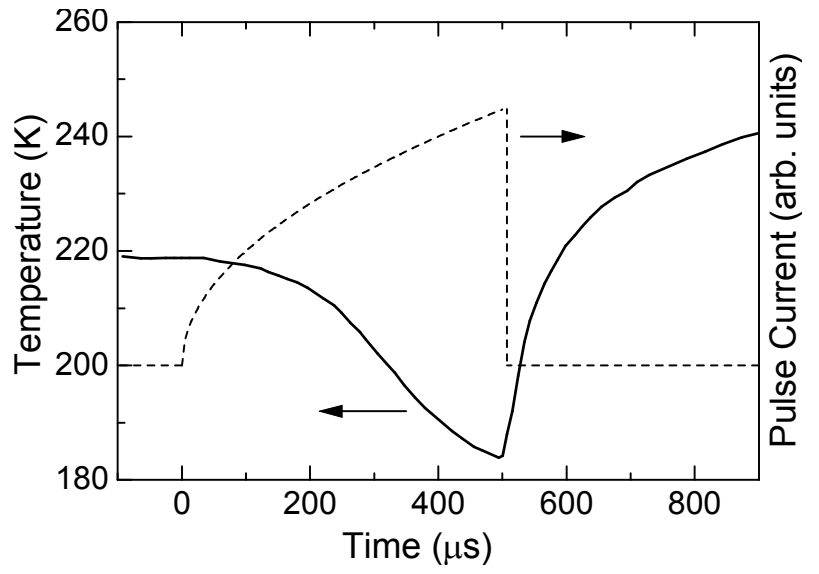

Figure 1: Calculated transient thermoelectric response [ 8$]$. A direct current is applied to a Peltier device, and the temperature at the cold end is kept at $220 \mathrm{~K}$ for $t<0$. Then a current pulse shown by the dashed curve is added, which significantly decreases the cold-end temperature down to $180 \mathrm{~K}$ at $500 \mu \mathrm{s}$.

sponse of a system with a time resolution ranging from femto- to pico-seconds. Since the electric field intensity of the incident laser pulse can be very large, one can observe various truly non-equilibrium phenomena such as photoinduced phase transitions [7]. These are quite interesting from the viewpoint of fundamental science, but the time scale is too short for thermoelectrics. In fact, after a "long" 95 time (say, $1 \mu \mathrm{s}$ ), absorbed photon energies are dissipated into heat to warm up the sample.

Much slower transient phenomena can effectively work in electronic refrigeration. Miner et al. [9] have proposed that the temperature drop of a Peltier device can be en- ${ }^{100}$ hanced by a factor of two by applying a short current pulse of $0.1-0.5 \mathrm{~ms}$ in addition to a direct current. The mechanism is that at the cold end, the Peltier refrigeration occurs instantaneously, but the Joule heat generated in the center of the device reaches late. Thronhauser et al. $[\bar{z}]^{105}$ have shown a simulation for the transient cooling as shown in Fig. Ш. A direct current is applied in the device in advance, and the temperature at the cold end is initially kept at $220 \mathrm{~K}$. Then a current pulse shown by the dashed curve is additionally applied, and the calculated temperature is ${ }^{110}$ plotted by the solid curve. The temperature reaches down to $182 \mathrm{~K}$ at $500 \mu \mathrm{s}$, which means $\Delta T$ increases from 80 to

so $118 \mathrm{~K}$. Since $\Delta T$ is directly proportional to $Z T$, the result in Fig, $\mathbf{W}$ indicates that an effective $Z T$ value increases by $50 \%$. After the pulse is turned off, the temperature goes up to $240 \mathrm{~K}$ owing to the Joule heat arriving late at the cold end. Later Yan and Malen [10] has experimentally ${ }_{115}$ 85 observed that the efficiency in such a transient mode can be enhanced by a factor of five.

A second example is a non-dissipative transport such as ballistic transport in nano-structured materials. Humphrey and Linke [II] have theoretically proposed a re-120 90 versible thermoelectric nano-device, and have shown that the efficiency equals the Carnot efficiency. Unfortunately, this device works only in open circuit conditions, and the

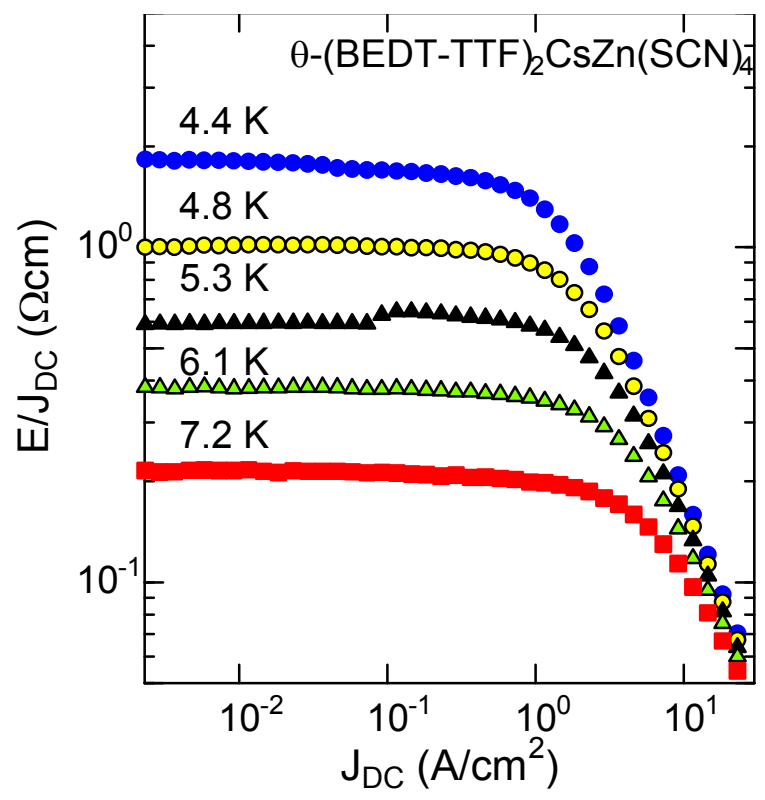

Figure 2: The resistivity of $\theta-(\mathrm{BEDT}-\mathrm{TTF})_{2} \mathrm{CsZn}(\mathrm{SCN})_{4}$ plotted as a function of current density.

power (work per unit time) is zero. Wu et al. [एँ2] have observed anomalous enhancement of the power factor $S^{2} / \rho$ in an InAs nanowire device, which is ascribed to the interference of the propagating electrons. Another type of nondissipative transport is the thermionic emission, a tunneling phenomenon of a thermally excited electron. Mahan and Woods [1.3] have proposed a thermionic refrigerator using a multilayer film, and Shakouri and his coworkers [14, 15] have made thermionic devices using semiconductor superlattices.

The non-equilibrium state we have studied thus far is a non-equilibrium steady state (NESS) - yet another nonequilibrium state, where the system is in a steady state with constant flows of particle and energy [16]. NESS has been regarded as a most simplified model for biological systems in the sense that it mimics homeostasis with exchanging substances and/or energies against the surroundings [17]. In the next sections, we will show anomalous thermoelectric properties in NESS.

\section{Thermoelectrics in high current density}

Non-ohmic conduction is a typical example for NESS, and among such systems we have focused on nonlinear conduction phenomena in strongly correlated electrons. As is generally agreed, strong correlation is a fertile source of electronic phase transitions such as high-temperature superconductivity in copper oxides, colossal magnetoresistance in manganese oxides, and multipole ordered states in heavy fermion intermetallics. Since some of such ordered states are susceptible to external impetus, we have searched for an ordered state susceptible to external electric field. 


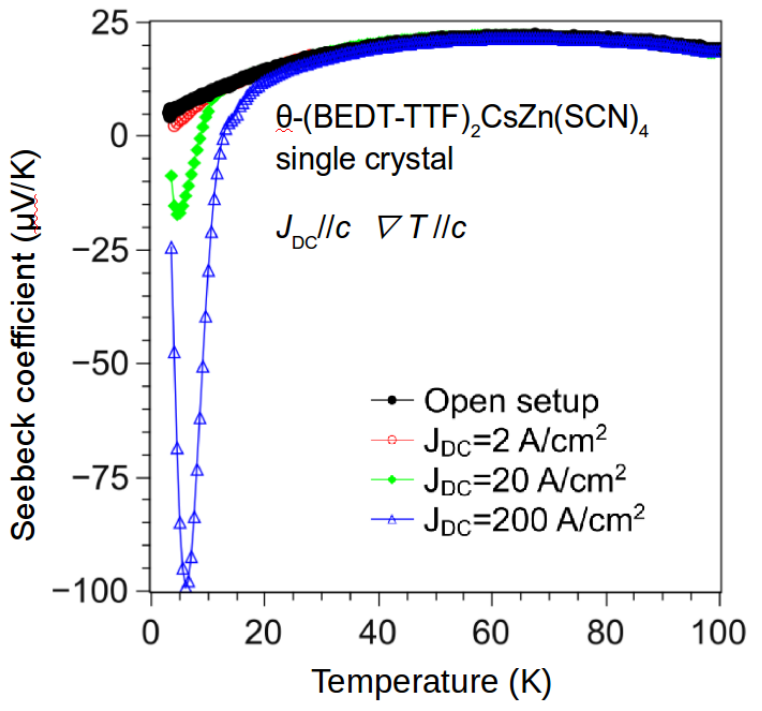

Figure 3: The Seebeck coefficient of $\theta-(\mathrm{BEDT}-\mathrm{TTF})_{2} \mathrm{CsZn}(\mathrm{SCN})_{4}$ plotted as a function of temperature with various de currents [ [25]. When the nonlinear conduction starts above $J_{d c}=2 \mathrm{~A} / \mathrm{cm}^{2}$, the Seebeck coefficient is largely enhanced with the sign change.

Eventually we discovered giant nonlinear conduction in an organic salt $\theta-(\mathrm{BEDT}-\mathrm{TTF})_{2} \mathrm{CsZn}(\mathrm{SCN})_{4}$ [18], as shown in Fig. $\boldsymbol{\nabla}$, and ascribed this to the suppression of $f_{160}$ the charge ordering by external current [10]. The charge order is one of the ordered states in strongly correlated electrons, in which conduction electrons form a lattice in order to minimize the Coulomb repulsion [20]. Since external current introduces electrons and holes to the sys- ${ }_{165}$ tem, it adversely affects the insulating nature of charge ordering. Ajisaka et al. [2] theoretically calculated this situation, and found that the external current reduces the -order gap. Other theoretical approaches have been reported to explain the nonlinear conduction of this com-170 pound $[22,[23]$. We further found that this organic salt exhibited a current oscillation with a dc voltage bias [24]. Such an oscillation is an intrinsically non-equilibrium phenomenon, and we named this salt "organic thyristor" [IT.

Figure 3 shows the Seebeck coefficient of $\theta-(B E D T-175$ $\mathrm{TTF})_{2} \mathrm{CsZn}(\mathrm{SCN})_{4}$ plotted as a function of temperature [25]. While the Seebeck coefficient for $J_{\mathrm{DC}}=0$ and 2 $\mathrm{A} / \mathrm{cm}^{2}$ is positive at all the temperatures, it changes the with a largely enhanced magnitude for 20 and 200 $\mathrm{A} / \mathrm{cm}^{2}$ below $10 \mathrm{~K}$. Since the nonlinear conduction also ${ }_{180}$ starts for $J_{\mathrm{DC}}>1 \mathrm{~A} / \mathrm{cm}^{2}$ in Fig. 2 , this enhanced Seebeck coefficient is ascribed to non-equilibrium effects. In this experiment, the effects of Joule heating are not perfectly controlled, but nevertheless we can conclude that such anomalous behaviour is due to non-thermal effects ${ }_{185}$ because the Seebeck coefficient for $J_{\mathrm{DC}}=0$ is positive up to $100 \mathrm{~K}$.

This behaviour is qualitatively similar to the See-

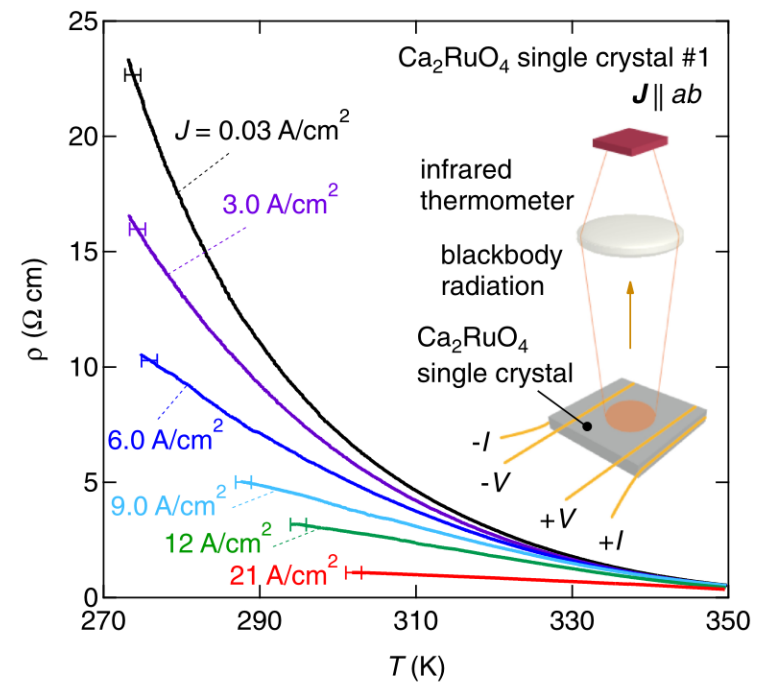

Figure 4: The resistivity of $\mathrm{Ca}_{2} \mathrm{RuO}_{4}$ plotted as a function of temperature with various dc currents.

(CDW) [26]. CDW is an ordered state of conducting electrons, where a charge density is sinusoidally modulated with a modulation period of $2 k_{F}$ through electron-phonon interaction, where $k_{F}$ is the Fermi wavenumber. Since $2 k_{F}$ is generally incommensurate with the lattice period, the phase of CDW is essentially independent of the lattice periodicity. Thus it can move relatively to the background lattice against a small electric field without dissipation, as is called "sliding motion" of CDW. Kriza et al. [27] found that the Seebeck coefficient of the CDW material $\mathrm{Rb}_{0.3} \mathrm{MoO}_{3}$ changes its sign above the threshold electric field where CDW starts to slide. We notice that the suppression of charge ordering is essentially different from the sliding CDW, and the same explanation unlikely to apply for the organic thyristor. Anyhow, external electric fields improve the thermoelectric performance in charge ordered materials and CDW materials.

Since the organic thyristor shows nonlinear conduction below around $10 \mathrm{~K}$, we have searched for a material showing nonlinear conduction at much higher temperature, preferably above room temperature. In the course of such material hunting, we have arrived at the Mott insulator $\mathrm{Ca}_{2} \mathrm{RuO}_{4}$; this particular oxide undergoes a metalinsulator transition at around $360 \mathrm{~K}$ [28, 2.9], and a low external pressure easily breaks the low-temperature insulating state [30]. Nakamura et al. [3] have recently discovered a small electric field also breaks the insulating state.

Making full use of advantages in room-temperature measurement, we have successfully controlled the sample temperature using black body radiation, and simultaneously observed the nonlinear conduction in isothermal conditions with various external current densities [32]. Figure $\mathrm{G}$ shows the nonlinear conduction of a single crystal of $\mathrm{Ca}_{2} \mathrm{RuO}_{4}$. We should emphasize that the temperature 


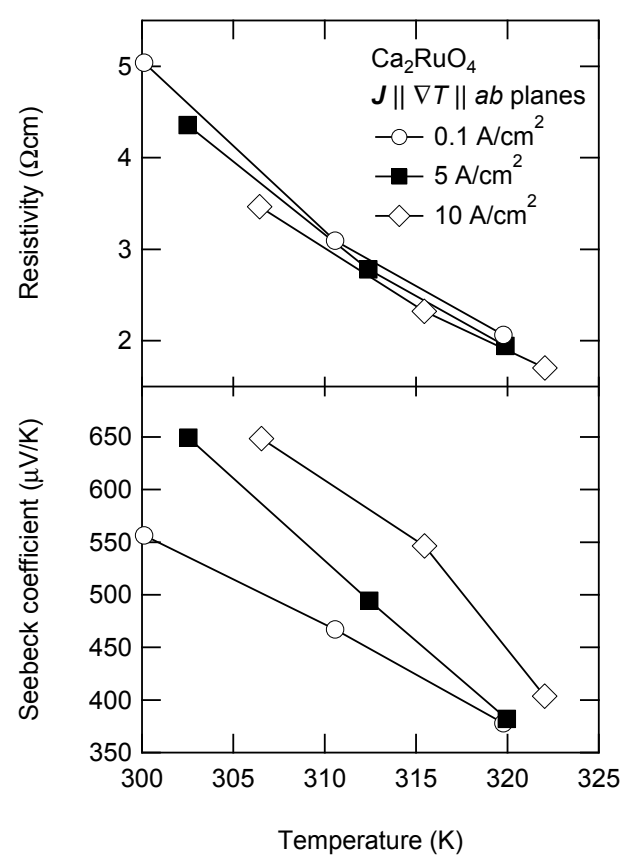

Figure 5: The Seebeck coefficient of $\mathrm{Ca}_{2} \mathrm{RuO}_{4}$.

is the sample temperature measured with an infrared pyrometer, as is schematically drawn in the inset of Fig. 国. With increasing current density, the resistivity systematically and smoothly decreases. Since the temperature is carefully controlled to be well below $T_{\mathrm{MI}}=360 \mathrm{~K}$, the samof metallic phase above $T_{\mathrm{MI}}$ likely generates.

Using the same technique, we have observed the Seebeck coefficient in various external currents. Figures 1 (a) and (b) show the simultaneous measurement of resistivity ${ }_{240}$ densities. The resistivity slightly decreases with external current density which is qualitatively similar to the data

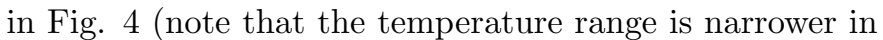
Fig. 回). In contrast, the Seebeck coefficient is significantly ${ }_{245}$ enhanced by $50-100 \mu \mathrm{V} / \mathrm{K}$, which cannot be ascribed to a simple self heating. Such enhancement may be related to hot carriers in semiconductors [3.3], but $\mathrm{Ca}_{2} \mathrm{RuO}_{4}$ is a poor conductor with strong electron-phonon coupling in which the electron temperature should equal the lattice ${ }_{250}$ temperature.

As mentioned in the introduction, a good thermoelectric material shows low resistivity and large Seebeck coefficient. In this respect, the external current and/or external electric field is a good option for good non-equilibrium ${ }_{255}$ thermoelectrics. Particularly when the sign changes with electric current density as in the case of the organic thyristor, we can make a p-n junction by using one kind of material with a bottleneck geometry, as is schematically drawn in Fig. [6.

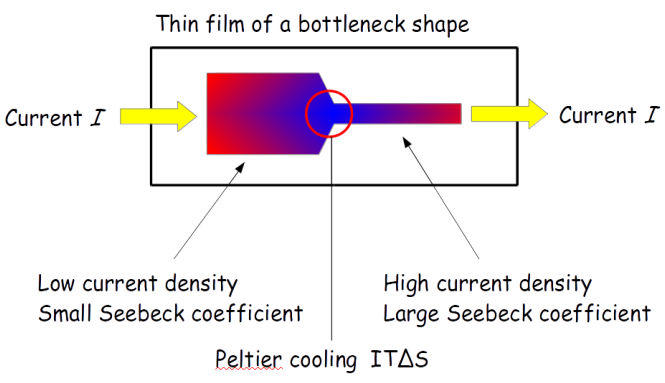

Figure 6: A nonlinear thermoelectric device with a bottle-neck geometry.

\section{Photo-thermoelectrics}

In this section we will discuss the thermoelectric transport under constant illumination of light. When a photon energy is larger than the band gap of a semiconductor, it is absorbed to generate an electron-hole pair. When light 25 is continuously illuminated, the system relaxes to a steady state where the creation rate of electron-hole pairs balances the recombination rate. We think that this is yet another non-equilibrium steady state, for photon energies much larger than the thermal energy are absorbed and dissipated steadily.

The photo-excited electron-hole pairs can contribute to charge transport, known as photo-transport. A most famous example is the photovoltaic phenomenon (solar cell), in which the photo-excited electrons and holes are spatially separated by the internal electric field existing at the p-n junction. In contrast, the mobility of electrons and holes is largely different in most materials, and onekind of carrier with higher mobility can be effectively introduced by the light illumination, which is called photodoping. Photo-conduction is an additional contribution of the photo-doped carriers to the electrical conduction.

If we regard photo-illumination as a means of carrier doping, we should revisit all the transport parameters as a function of photo-doped carrier concentration. For example, the photo-Seebeck effect is the Seebeck effect shown by the photo-doped carriers. Although the first observation of the photo-Seebeck effect was reported in the mid 50 's [34], there have been very few reports on the photoSeebeck measurements [35, 36]. Thus we had to newly establish measurement procedure with recently available equipment such as a light-emitting diode (LED) [37].

We have observed the photo-Seebeck effect in $\mathrm{ZnO}$ [38], $\mathrm{PbO}$ [3.9], $\mathrm{Pb}_{2} \mathrm{CrO}_{5}$ [40] and $\mathrm{ZnS}$ [41]. Among them, we think that $\mathrm{ZnO}$ is the most promising material, not only because it is a binary oxide with abundant elements, but also because it is a high-mobility semiconductor when carefully prepared [42]. When we observed the photoconductivity and the photo-Seebeck effect in a $\mathrm{ZnO}$ single crystal, we found that the crystal was much thicker than the skin depth (penetration depth) of the ultraviolet light we measured. Generally, when the photon energy 


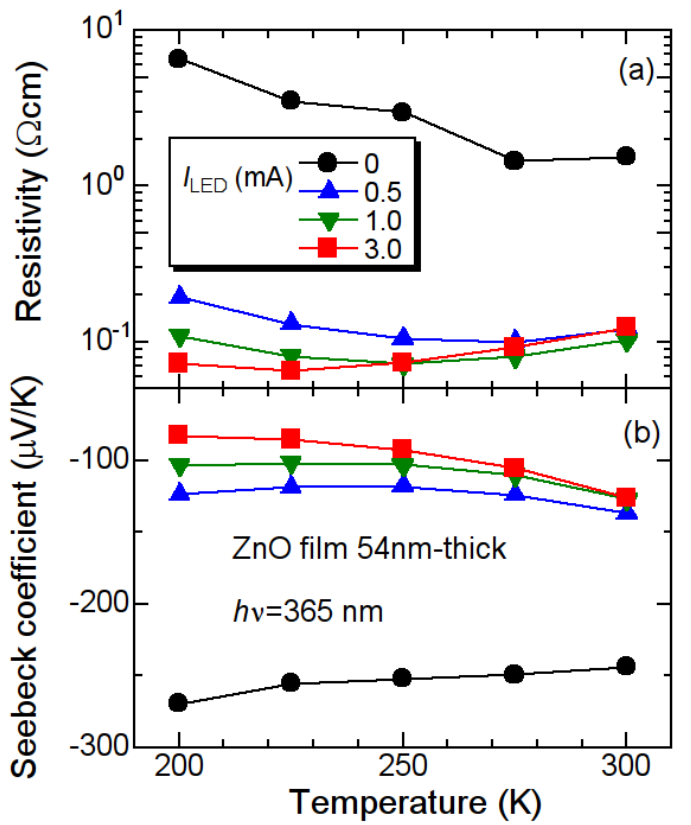

Figure 7: The photo-Seebeck coefficient of $\mathrm{ZnO}$ thin film.

of an incident light is larger than the band gap of an insulator/semiconductor, the light is immediately absorbed at the surface. In this respect, the photo-Seebeck effect and photo-thermoelectrics is a surface phenomenon within the skin depth (typically, of the order of 10-100 nm). To extract intrinsic values of the photo-excited area, we employed a simple two layer model [38, 3.9]. This analysis should be examined by measuring thin film samples with the film thickness smaller than the skin depth.

We have prepared a high-quality epitaxial thin film of $\mathrm{ZnO}$ on $\mathrm{Y}_{2} \mathrm{O}_{3}$-stabilized $\mathrm{ZrO}_{2}$ substrates. The detailed growth conditions were written elsewhere [43]. The film thickness was evaluated to be $52 \mathrm{~nm}$ from the Laue oscilation in the x-ray diffraction pattern, which is comparable with the skin depth for a wave length of $365 \mathrm{~nm}$. Figure $\mathrm{I}$ (a) shows the resistivity plotted as a function of temperature with various light intensities. The value of the input current of LED ( $\left.I_{\mathrm{LED}}\right)$ corresponds to the light intensity zero for the dark state and $3.0 \mathrm{~mA}$ for the maximum illumination). Owing to the experimental constraints, we were unable to calibrate the light intensity precisely, but $I_{\text {LED }}=1.0 \mathrm{~mA}$ roughly corresponds to a photon flux of $10^{14}$ photons $/ \mathrm{cm}^{2} \mathrm{~s}$ judging from a different experiment [39]. As shown in Fig. $\square\left(\right.$ a), the resistivity for $I_{\mathrm{LED}}=0 \mathrm{~mA}$ is of the order of $1 \Omega \mathrm{cm}$ at room temperature, and slightly increases with decreasing temperature. This indicates that the doping level of the $\mathrm{ZnO}$ film is in the lightly doped region, perhaps owing to uncontrolled non stoichiometry at the grain boundaries. With light illumination, the resistivity suddenly drops to $0.1 \Omega \mathrm{cm}$ at $I_{\mathrm{LED}}=0.5 \mathrm{~mA}$, and almost saturates for larger $I_{\mathrm{LED}}$. We should note that the temperature dependence of the resistivity changes from

non-metallic at dark to metallic at $I_{\mathrm{LED}}=3.0 \mathrm{~mA}$, which 295 degenerate region.

Figure $\square(\mathrm{b})$ shows the Seebeck coefficient for various values of $I_{\text {LED }}$. Similarly to the resistivity, the Seebeck coefficient at dark slightly increases in magnitude with weaker than $T^{-1}$, suggesting that the doping level is in the lightly doped region. With light illumination, the magnitude decreases from 250 to $130 \mu \mathrm{V} / \mathrm{K}$ at $300 \mathrm{~K}$. Also, the temperature dependence of the Seebeck coefficient is sample is in the degenerate region. Using the nearly free electron model, the Seebeck coefficient can be given as $S=\pi^{2} k_{B}^{2} T / 3 e \varepsilon_{F}$, where $\varepsilon_{F}$ is the Fermi energy. From the obtained $\varepsilon_{F}$, we evaluate the carrier concentration to the same as the value that we evaluated in the $\mathrm{ZnO}$ single crystal induced by light illumination using the two-layer model. By combining the resistivity of $0.06 \Omega \mathrm{cm}$ at $230 \mathrm{~K}$ and the carrier concentration of $10^{19} \mathrm{~cm}^{-3}$, we estimate 5 the mobility of the $\mathrm{ZnO}$ film to be $1 \mathrm{~cm}^{2} / \mathrm{Vs}$.

Here we will discuss the feasibility and prospect of photo-thermoelectrics. At present, the carrier concentration of $10^{19} \mathrm{~cm}^{-3}$ seems to an upper limit for the $\mathrm{ZnO}$ film sample. This is close to an optimum value for thermoelectric semiconductors, and consequently we can say that photo-doping is a good option to carry out carrier doping in thermoelectric materials. According to Ref. [42], the best mobility of $\mathrm{ZnO}$ is around $150 \mathrm{~cm}^{2} / \mathrm{Vs}$ at 300 $\mathrm{K}$, which gives a resistivity of $0.4 \mathrm{~m} \Omega \mathrm{cm}$ and a Seebeck coefficient of $130 \mu \mathrm{V} / \mathrm{K}$ at room temperature. These values are close to those in a $\mathrm{Na}_{x} \mathrm{CoO}_{2}$ single crystal [44], and are comparable to those for $\mathrm{Bi}_{2} \mathrm{Te}_{3}$. Thus we optimistically conclude that carefully prepared $\mathrm{ZnO}$ thin film can be used as an alternative to $\mathrm{Bi}_{2} \mathrm{Te}_{3}$ at room temperature. For example, we propose a $\mathrm{ZnO}$-coated window glass which can effectively cut ultraviolet light and generate a finite electricity through a temperature difference between inside and outside of the building.

\section{Summary and Future Prospects}

In this article, we have reported our attempts to find a better thermoelectric power generation using nonequilibrium steady states. First approach is to use nonlinear conduction in strongly correlated electrons. In this case, a large current density decreases resistivity by a factor of ten or more and enhances the magnitude of the Seebeck coefficient. Although the mechanism of the enhancement is yet to be explored, the thermoelectric properties are significantly improved just by increasing the external current. Another approach is the thermoelectrics using the photo-Seebeck effect. We have shown that the n-type photo-Seebeck effect in $\mathrm{ZnO}$ is useful, and the achievable values using high-quality films are comparable with conventional thermoelectric materials. In this sense, we pro- 
pose that the photo-doping is a good option for carrier Although our results are still far from practical ${ }^{410}$ goals, we believe that non-equilibrium states are worth exploring not only for fundamental sciences but also for novel functional materials.

\section{Acknowledgements}

The authors would like to thank Majed Abdel-Jawad, Hatsumi Mori, Takehiko Mori, Ayaka Horikawa, Masaru Fujita, Yasuo Nishina, Fumihiko Nakamura, Yoshio Nogami, Shigeru Ajisaka, and Shin Nakamura for collaboration and fruitful discussion. This work was parially supported by ALCA, Japan Science and Technology Agency, by The Mitsubishi Foundation, and by a Grantin-Aid for Scientific Research from the Ministry of Education, Culture, Sports, Science and Technology, Japan (Nos. 26287064 and 26247060), and by the Network Joint

Research Center for Materials and Devices.

\section{References}

[1] I. Terasaki, in: P. B. F. Kamimura (Ed.), Comprehensive Semiconductor Science and Technology, Elsevier, Amsterdam, 2011, pp. 326 - 358. doi:10.1016/B978-0-44-453153-7.00070-5.

[2] G. Mahan, in: H. Ehrenreich, F. Spaepen (Eds.), Solid State ${ }^{441}$ Physics, volume 51 of Solid State Physics, Academic Press, 1997, pp. 81 - 157. doi:10.1016/S0081-1947(08)60190-3.

[3] G. J. Snyder, E. S. Toberer, Nat. Mater. 7 (2008) 105-114. doi:10.1038/nmat2090.

375 [4] J. He, Y. Liu, R. Funahashi, J. Mater. Res. 26 (2011) 1762-1772.445 doi:10.1557/jmr.2011.108.

[5] G. Casati, C. Mejía-Monasterio, T. Prosen, Phys. Rev. Lett. 101 (2008) 016601. doi:10.1103/PhysRevLett.101.016601.

[6] H. Aoki, N. Tsuji, M. Eckstein, M. Kollar, T. Oka, P. Werner, Rev. Mod. Phys. 86 (2014) 779-837. doi:10.1103/RevModPhys. ${ }^{450}$ 86.719 .

[7] S. Koshihara, J. Phys.: Conf. Ser. 21 (2005) 7. doi:10.1088/ 1742-6596/21/1/002.

[8] T. Thonhauser, G. D. Mahan, L. Zikatanov, J. Roe, Appl. Phys. Lett. 85 (2004) 3247-3249. doi:10.1063/1.1806276.

[9] A. Miner, A. Majumdar, U. Ghoshal, Appl. Phys. Lett. 75 (1999) 1176-1178. doi:10.1063/1.124634.

[10] Y. Yan, J. A. Malen, Energy Environ. Sci. 6 (2013) 1267-1273. doi:10.1039/C3EE24158K.

[11] T. E. Humphrey, H. Linke, Phys. Rev. Lett. 94 (2005) 096601.460 doi:10.1103/PhysRevLett.94.096601.

[12] P. M. Wu, J. Gooth, X. Zianni, S. F. Svensson, J. G. Gluschke, K. A. Dick, C. Thelander, K. Nielsch, H. Linke, Nano Letters 13 (2013) 4080-4086. doi:10.1021/nl401501j.

[13] G. D. Mahan, L. M. Woods, Phys. Rev. Lett. 80 (1998) 4016-405 4019. doi:10.1103/PhysRevLett.80.4016.

[14] A. Shakouri, J. E. Bowers, Appl. Phys. Lett. 71 (1997) 12341236. doi:10.1063/1.119861.

[15] A. Shakouri, P. Abraham, C. LaBounty, J. Bowers, in: Thermoelectrics, 1998. Proceedings ICT 98. XVII International Conference on, pp. 218-220. doi:10.1109/ICT.1998.740356.

[16] S.-i. Sasa, H. Tasaki, J. Stat. Phys. 125 (2006) 125-224. doi:10. 1007/s10955-005-9021-7.

[17] F. Ritort, Nonequilibrium Fluctuations in Small Systems: From Physics to Biology, John Wiley and Sons, Inc., 2008, pp. 31-123. doi: $10.1002 / 9780470238080$. ch2

[18] K. Inagaki, I. Terasaki, H. Mori, T. Mori, J. Phys. Soc. Jpn. 73 (2004) 3364-3369. doi:10.1143/JPSJ.73.3364.
[19] F. Sawano, I. Terasaki, H. Mori, T. Mori, M. Watanabe, N. Ikeda, Y. Nogami, Y. Noda, Nature 437 (2005) 522-524. doi:10.1038/nature04087.

[20] H. Seo, Journal of the Physical Society of Japan 69 (2000) 805820. doi:10.1143/JPSJ.69.805.

[21] S. Ajisaka, H. Nishimura, S. Tasaki, I. Terasaki, Prog. Theor. Phys. 121 (2009) 1289-1319. doi:10.1143/PTP.121.1289.

[22] S. Nakamura, Prog. Theor. Phys. 124 (2010) 1105-1114. doi:10. 1143/PTP.124.1105.

23] Y. Tanaka, K. Yonemitsu, Phys. Rev. B 83 (2011) 085113. doi:10.1103/PhysRevB.83.085113.

[24] T. Suko, I. Terasaki, H. Mori, T. Mori, Materials 3 (2010) 2027 2036. doi:10.3390/ma3032027.

[25] M. Abdel-Jawad, I. Terasaki, T. Mori, H. Mori, J. Phys. Soc. Jpn. 84 (2015) 033707. doi:dx.doi.org/10.7566/JPSJ. 84. 033707 .

[26] G. Grüner, Rev. Mod. Phys. 60 (1988) 1129-1181. doi:10.1103/ RevModPhys.60.1129.

[27] G. Kriza, A. Jánossy, L. Forró, Phys. Rev. B 41 (1990) 54515454. doi:10.1103/PhysRevB.41.5451.

[28] S. Nakatsuji, Y. Maeno, Phys. Rev. Lett. 84 (2000) 2666-2669. doi:10.1103/PhysRevLett.84.2666.

[29] S. Nakatsuji, Y. Maeno, Phys. Rev. B 62 (2000) 6458-6466. doi:10.1103/PhysRevB.62.6458.

[30] F. Nakamura, T. Goko, M. Ito, T. Fujita, S. Nakatsuji, H. Fukazawa, Y. Maeno, P. Alireza, D. Forsythe, S. R. Julian, Phys. Rev. B 65 (2002) 220402. doi:10.1103/PhysRevB. 65.220402.

[31] F. Nakamura, M. Sakaki, S. Yamanaka, Y. Tamaru, T. Suzuki, Y. Maeno, Sci. Rep. 3 (2013) 2536. doi:10.1038/srep02536.

[32] R. Okazaki, Y. Nishina, Y. Yasui, F. Nakamura, T. Suzuki, I. Terasaki, J. Phys. Soc. Jpn. 82 (2013) 103702. doi:10.7566/ IPS.J. 8\%. 1037(0)

[33] M. Zebarjadi, K. Esfarjani, A. Shakouri, Appl. Phys. Lett. 91 (2007) 122104. doi:10.1063/1.2785154.

[34] J. Tauc, Czech. Phys. J. 5 (1955) 528-535. doi:10.1007/ BF01687219.

[35] J. G. Harper, H. E. Matthews, R. H. Bube, J. Appl. Phys. 41 (1970) 3182-3184. doi:10.1063/1.1659387.

[36] H. B. Kwok, R. H. Bube, J. Appl. Phys. 44 (1973) 138-144. doi:10.1063/1.1661848.

37] I. Terasaki, R. Okazaki, P. Mondal, Y.-C. Hsieh, Mater. Renew. Sustain. Energy 3 (2014). doi:10.1007/s40243-014-0029-2.

[38] R. Okazaki, A. Horikawa, Y. Yasui, I. Terasaki, J. Phys. Soc. Jpn. 81 (2012) 114722. doi:10.1143/JPSJ.81.114722.

[39] P. S. Mondal, R. Okazaki, H. Taniguchi, I. Terasaki, J. Appl. Phys. 114 (2013) 173710. doi:10.1063/1.4829460.

[40] P. S. Mondal, R. Okazaki, H. Taniguchi, I. Terasaki, J. Appl. Phys. 116 (2014) 193706. doi:10.1063/1.4902248.

[41] Y. Shiraishi, R. Okazaki, H. Taniguchi, I. Terasaki, Jpn. J. Appl. Phys. 54 (2015) 031203. doi:dx.doi.org/10.7567/JJAP. 54.031203

[42] S. Akasaka, A. Tsukazaki, K. Nakahara, A. Ohtomo, M. Kawasaki, Jpn. J. Appl. Phys. 50 (2011) 080215. doi:10. $1143 /$ JJAP. 50.080215.

[43] H. Ohta, K.-i. Kawamura, M. Orita, M. Hirano, N. Sarukura, H. Hosono, Appl. Phys. Lett. 77 (2000) 475-477. doi:http:// dx.doi.org/10.1063/1.127015.

[44] I. Terasaki, Y. Sasago, K. Uchinokura, Phys. Rev. B 56 (1997) R12685-R12687. doi:10.1103/PhysRevB.56.R12685. 